\title{
A Mobile Tool for In-Situ Prototyping
}

\author{
Marco de Sá \\ LaSIGE \& University of Lisboa \\ Luís Carriço \\ LaSIGE \& University of Lisboa \\ Sala 6.3.30, FCUL, 1749-016, Campo-Grande, Portugal Sala 6.3.30, FCUL, 1749-016, Campo-Grande, Portugal \\ marcosa@di.fc.ul.pt \\ Imc@di.fc.ul.pt
}

\begin{abstract}
Mobile interaction design introduces added challenges when compared with the usual design process for fixed technologies. In particular, it benefits greatly from the ability to take the design process out of the lab, creating, designing and evaluating applications within their natural usage context. However, this process requires new and deeply refined approaches to traditional techniques which are demanding and still avoided by designers. This paper presents a tool that overcomes some of these issues by offering means to support in-situ design. The tool supports prototyping of mobile applications and user interfaces on real scenarios, also providing evaluation features that allow the logging and analysis of usage information. We describe the tool's concept, goals and design implications, focusing, in particular, its out-on-the-field, in-situ design and prototyping features.
\end{abstract}

\section{Categories and Subject Descriptors}

H5.2. [Information interfaces and presentation] (e.g., HCI): User Interfaces - Evaluation/Methodology, Prototyping, Usercentered Design

\section{General Terms}

Design, Experimentation, Security, Human Factors.

\section{Keywords}

Mobile Prototyping, Usability Evaluation, Participatory Design.

\section{INTRODUCTION}

Mobile interaction design is a demanding process that introduces additional challenges that go beyond those of fixed/desktop computers. The diversity of usage contexts and more importantly, the ubiquity and extension of activities through various settings and environments pose new difficulties to designers while conceiving a User Interface that must remain usable at all times. Moreover, the specific characteristics that define mobile devices (e.g., small screens, lack of keyboard, weight, battery) in concert with specific and mixed interaction modalities, albeit extremely useful, must be coped with while designing usable applications in order to fulfil their full potential.

This problem has been gaining attention within the research community and various approaches have emerged, targeting different concerns and issues [8]. However, a global consensus that has been achieved seems to point the need to take the design process, in particular prototyping and evaluation, out of the lab, into the field, where realistic settings can be used [4,3,13,14]. In order to facilitate the process, recent results have been providing guidelines that suggest how to select appropriate scenarios,

Copyright is held by the author/owner(s).

MobileHCI’09, September 15 - 18, 2009, Bonn, Germany.

ACM 978-1-60558-281-8. material and techniques to support in-situ design [15]. In particular, main lessons suggest that besides using realistic settings, designers must aim at providing realistic usage experiences, making use of realistic prototypes [14]. In addition to resembling actual devices and emulating their characteristics, this approach promotes participatory design, emphasizing user engagement on the sketching process, and offers better support for field/in-situ evaluation. Still, despite the positive results and impact that these approaches have when designing for mobile devices; they require added effort from designers which often lead to their avoidance and, consequentially, poor or unusable applications. Additionally, analysis can rarely be done on-the-spot and for users, even realistic low-fidelity paper-based prototypes can become awkward to use which also hinder the design process.

This paper presents a tool that aims at tackling these issues by providing designers with support for in-situ prototyping and evaluation directly on mobile devices. The tool allows designers to create and refine mixed-fidelity prototypes and to collect usage data on the go, while users interact with the prototypes on actual mobile devices in the real world. We start by addressing current approaches that aim at solving similar issues and discussing the problems and solutions that motivated this work. We proceed with the description of the in-situ prototyping tool, providing the context and background detail that motivated its development, followed by case studies. Finally, we discuss the results and our tool's impact on the design process and delineate future research directions.

\section{RELATED WORK}

Design methods, techniques and tools for mobile devices are increasingly being addressed by researchers, leading to the appearance of different approaches for a wide range of problems $[4,14,15,8]$. Unsurprisingly, given their differences from desktop systems, most efforts have been directed towards prototyping and evaluation.

Regarding prototyping, new techniques and orientations, particularly for low-fidelity prototypes, have been introduced $[14,15]$. These suggest the need for more detailed and carefully built prototypes that offer resembling pictures of final solutions and their characteristics [14], even at very early stages [17, 18]. In fact, it has been shown in [15] that the adopted prototyping technique can be determinant during the consequent evaluation stages, allowing users to freely interact with them, improve them and use them on realistic settings without being misled [6]. Furthermore, to evaluate different details that might be relevant at different stages of prototyping, the concept of mixed prototyping has emphasized the need to create different prototypes to evaluate different dimensions of usability [12]. On these aspects, prototyping tools can play a paramount role, allowing designers to maintain their sketching and writing practices while creating 
prototypes that can actually be executed, giving users a more tangible and realistic feel of the future application.

DENIM [11] and SILK [10] are good examples of prototyping tools that give designers the ability to quickly create sketch-based prototypes and interact with them on the computer, also including the possibility of replacing drawn components with actual programmatic components. More recently, systems such as SketchWizard [2] or SUEDE [9] have also emerged, supporting new modalities and interaction modes such as pen-based input on the former and speech user-interfaces on the latter. Ex-A-Sketch [5] also allows designers to quickly animate sketches drawn on a whiteboard.

However, although these tools have very useful functionalities and features, and provide sketching and quick prototyping mechanisms, the ability to conduct such endeavours out on the field is still unavailable. Moreover, the integration and support for evaluation techniques that can be used ubiquitously is nonexistent and the available tools rarely address the specific needs of mobile devices. Nevertheless, the existing features, in particular the support for automatic Wizard-of-Oz prototypes and the ability to animate hand drawn sketches have shown very positive results.

As aforementioned, problems are felt again when evaluating the developed prototypes. Although some recent studies reflect an increasing amount of attention towards contextual evaluation, out of the lab, its relative inexistence contrasts with the importance and benefits it presents to mobile devices $[1,3,4,13]$. The reasons behind this lack of in-situ evaluation generally point the need for adjusted techniques or tools $[4,8]$ that can aid designers when providing users with realistic user experiences at early stages. In order to support these goals and to overcome existing limitations, we integrate the prototyping and evaluation features into one tool that can be used on real devices without depending on connections or frequent synchronizations.

\section{MOTIVATION}

The mobile in-situ prototyping tool discussed in this paper is part of a larger framework, which supports the design and evaluation of mobile multimodal applications with various fidelities [16]. Globally, the framework provides users and designers with features for the design of sketch-based or visual component-based prototypes. It is composed by a prototyping environment (available for desktop/laptop computers) which supports a visual, non-programmatic design of cards/screens that compose an interactive prototype. Prototypes can be created using images, scanned sketches or drawings, augmented with behaviour, defined by a set of triggers and conditions (e.g., if user clicks inside this area, jump to next screen) or using visual components (e.g., dropboxes, text-boxes, buttons, track-bars). The latter assume their usual behaviour (e.g., textbox allows for text input) but can also be augmented with additional conditions (e.g., if user exceeds 10 characters, pop a warning). The prototyping tool and the overall framework also support the evolution of the design concept by offering means to replace hand drawn elements with interactive components, moving to higher-fidelity prototypes.

Besides allowing designers/developers to quickly create prototypes materializing their visions and concepts, the framework's scope is extended to the usage of these prototypes in actual devices also focusing data collection and its analysis. Accordingly, once a prototype is created, it is stored in an XML file that can be copied into a mobile device. Here, a runtime environment recreates the prototype and allows end-users to freely interact with it, overcoming issues related to realism and even interactivity (e.g., the prototype can be used without a designer acting as the Wizard-of-Oz). At the same time, the runtime environment collects usage data through a configurable logging mechanism. Currently we have runtime environments for Windows, Windows Mobile, and PalmOS.

On the remainder of this paper, we address the mobile extension to the creation tool, which emerged from the need to adjust, update and create prototypes on-the-go, while using and testing them on real-life outdoor settings. We stress the requirements that were established because of the different platforms and highlight the features that were ported to the mobile version of the prototyping tool. The tool is available for Windows Mobile.

\section{ADJUSTMENTS AND NEW FEATURES}

The in-situ prototyping tool aims at supporting a set of techniques that have proven to enhance the design process of mobile user interfaces. These were previously applied and validated by the utilisation of the entire framework on various case studies [16]. However, given the positive results, and the need to carry-on with the design process in-situ, while testing the prototypes and when users were interacting with them, a mobile extension was required. Overall, the goal is to combine the advantages of rapid prototyping and adjustment of sketches on realistic settings and scenarios, with the added features that the digital medium can provide. Naturally, and given the underlying platform (e.g., PDAs, SmartPhones) and its screen and processing limitations, only the most important features could be included. Nevertheless, care was taken to maintain the easy, non-programmatic design approach, endowing end-users to participate on the prototype's design and adjustment as well.

Architecturally, the tool is embedded into the runtime environment and can be instantiated through the push of a button. Running mode and editing mode can be alternated at will. Once the editing mode is selected, every element that composes a screen (e.g., picture-box, drop-box, button) is copied into a invisible container which allows users to resize it, move it around the screen, edit its content and delete it. To add any new element, users must select it from the options menu. Once a new element is added, changes can be easily done through the previous mechanism or by long pressing (pressing for more than 2 seconds) the element, which displays a contextual menu (Figure 2, left).

Contrarily to the desktop version, in which several screens can be edited simultaneously and visually manipulated within a work area [16], the mobile version only allows users to edit one screen at each time (following the paper-based analogy, each screen depicts a card). Nevertheless, screens can be easily navigated through a side pane, which allows users to see all the screens that compose the prototype and directly select the desired one (figure 1, left). Moreover, the same method is used to define the navigation restrictions. Figure 1 on the left shows a user linking an interactive "Click Area" on a sketch-based prototype to the target screen. In summary, this will allow the end-user to interact with the sketch, taping the transparent click area, placed on top of a drawn button, and navigating through the prototype (Figure 1, right). Click areas are also marked with a tab number which allows users to navigate through them by using a keypad or a 
joystick. Interactive areas can also be highlighted if selected by the designer, alerting users to interactive areas within a sketch.

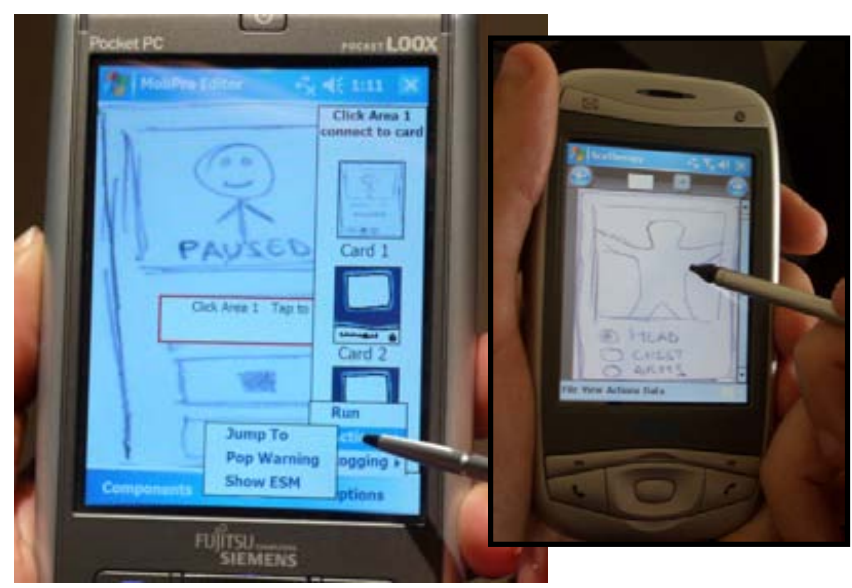

Figure 1. Left) Mobile Prototyping Environment. Right) EndUser interacting with a low-fidelity, sketch-based prototype.

New prototypes can also be created from scratch. On this facet, screen editing is maintained and screens can be added to the prototype by selecting the "add new screen" option.

Limitations of the mobile editor include the inability to use textto-speech when creating multimodal elements and the definition of gesture-based triggers. Nevertheless, audio input/output elements and video/image input/output elements are still available. Additionally, because of the ability to create and adjust prototypes on the context of use, new or adjusted features also emerged:

- $\quad$ In-Situ Sketch Augmentation - By providing the option to augment sketches with behaviour, the tool allows users to maintain their sketching procedures (e.g., drawing a UI on a sheet of paper or card) and to turn it into an interactive prototype. For instance, if the device includes a camera, the user can easily capture a photo of a hand-drawn sketch, import it to the editor and define interactive areas within it or place software elements (e.g., buttons) on top of it.

- $\quad$ Card Sorting - Since prototypes can be edited directly on the mobile device, while interacting with it, contextual card sorting is supported. This is an effective technique that relies on end-users to arrange the sequence or organisation of different cards (representing the UI screens) according to their preferences or needs, within and depending on different contexts of use [15]. Following the same mechanisms described previously, end-users can also easily change the screens sequence, sorting them as they deem adequate.

- $\quad$ Card Tagging and Annotation - Using text-box elements or audio recording elements allows users to tag and annotate cards on the device. Each card can be tagged and annotated at any given time, allowing users, or designers, to store thoughts, user reactions or adjustments to that card/screen.

- Data Gathering Configuration - Although not the focus of this paper, it is worth mentioning that the mobile tool also includes the ability to configure the data gathering techniques that it integrates. With the same mechanisms used to build software prototypes, users are able to create
Experience Sampling Method (ESM) [1] (figure 3, left) questionnaires that can be shown and popped according to different conditions (e.g., after 1 minute using one card). For the logging mechanism, data granularity (e.g., every event, taps on the screen, typed characters) can be adjusted and a player which allows users to review the stored logs and user's interaction with the prototype, is also provided.

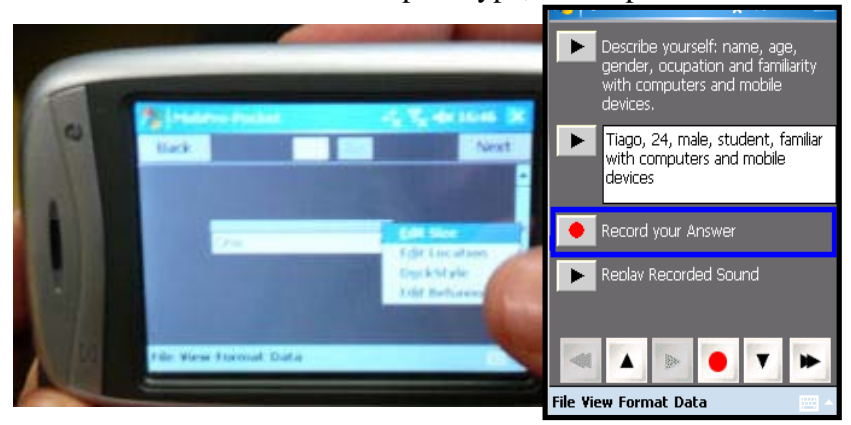

Figure 2. Left) Designer editing a text-box directly on the mobile device. Right) High-fidelity prototype with labels, textboxes, buttons and audio recording elements.

\section{CASE STUDIES AND RESULTS}

The entire framework has been successfully used during the design process of several applications targeting mobile devices [16]. Still, the inclusion of a mobile editor has propelled the emergence of new design implications and opportunities that were previously unavailable. By introducing functionalities that allow designers to adjust prototypes on-the-spot, the tool has promoted creativity and end-user participation. During field tests, when faced with new situations and requirements, designers frequently created additional sketches or adjusted existing ones to match the needs of that situation. Figure 3 shows a designer taking a photograph of a sketch, created in-situ, while designing a botanical cataloguing tool. The photographs were immediately imported into a prototype, interactive areas were added and different versions of the prototype were tested on-the-spot.

During one particular experience targeting mobile psychotherapy, given the ethical restraints that prevented the involved designers to assist to therapy sessions, the tool was actually used to prototype by therapists and patients while using the devices during therapy sessions (mostly adjustments to initial prototypes). Moreover, annotations were added describing where issues were detected and the end-users' opinions on the user experience.

The visual creation and prototyping process, using the same devices that are the target of the application, promoted participatory design. End-users were actively involved both in the sketching process (most of the times using simple the drawing software available on the device) and on the actual construction and arrangement of the sequence and navigational constraints that bring the prototypes to life. The multimodal elements of software prototypes were also frequently used to test auditory icons and their effectiveness in crowded locations. This was particularly relevant during a field test (for a digital book player prototype) with a visually impaired user who was able to provide input by recording his thoughts and testing the prototype's audible content.

Designers were particularly pleased with the ability to immediately correct and test adjustments that were made to button 
sizes and location, evaluating their adequateness to different situations (e.g., walking, seating). Questionnaires that were responded after the first experiences showed a very positive response (over $80 \%$ acceptance) toward the tool and the vast majority (89\%) of the designers stated that they would use the tool during their own design experiences.

End-user responses were also extremely favourable. The possibility of seeing the design concepts come to life on actual devices without being followed by anyone, was very appreciated.

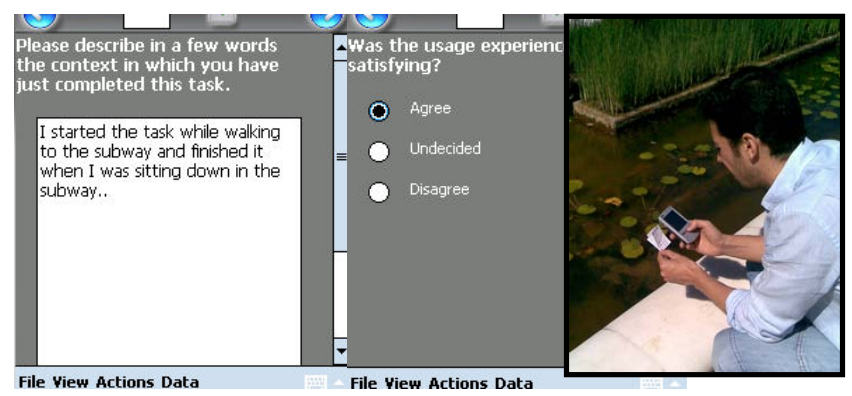

Figure 3. Left) ESM questionnaires. Right) End-user taking photos of paper sketches for an in-situ prototyping experience.

Globally, design concepts emerged on-the-spot, triggered by the requirements that surfaced while testing several approaches and design alternatives in the realistic settings. This also enabled the usage of the framework for probing purposes [7], promoting experimentation and on-the-fly design of new solutions for the context in which users interacted with the tool. By offering the ability to design in-situ, collecting data and adjusting designs to the current context, overall results were much more positive than previous experiences with traditional paper-based prototypes [15].

\section{CONCLUSIONS}

The use of realistic prototypes that can be used on actual devices and on real world situations has proved to have great impact on the design of mobile applications [16]. Now, the ability to overcome the challenges of in-situ design and evaluation, through the use of mobile devices and their potentialities clearly provided benefits at various levels, namely allowing designers to build and test design concepts on-the-go. This paper presented a tool that materialized and enhanced these benefits, facilitating designer's activities while conduction real world design and evaluation, but also providing end-users with realistic prototypes that can be used and freely interacted with (without the designer's presence) on real devices.

Besides propelling creativity, on-the-spot adjustments and experimentation of design concepts and approaches, the mobile editor has also supported and fomented user participation on the design process. The hands-on approach that the tool offers, in conjunction with the real-world settings in which it can be used, triggered user's imagination while focusing the context and supporting immediate testing of the developed prototypes.

Globally, it provided much shorter design cycles, which, when combined with the realistic usage experience, provided better results. Moreover, the integration of multimodalities that support evaluation augmented the gathered data and proved to be less intrusive while providing means to gather data passively and actively (e.g., voice-based input ESM, capturing images and video clips of the surrounding environment). These also allowed for the evaluation of the prototypes' accessibility levels. In concert with these abilities, the possibility of analysing, even during evaluation sessions, interaction data, further reduces design cycles, bringing closer the design and testing stages.

Future work directions include overcoming of the stated limitations, especially gesture recognition on the prototypes, and the possibility of geo-tagging events triggered while users are interacting with the prototypes (e.g., user registered this thought or had low accuracy toward a button in this context/location).

\section{REFERENCES}

1. Consolvo, S. and M. Walker, Using the Experience Sampling Method to Evaluate Ubicomp Applications. IEEE Pervasive Computing, 2003. 2(2): p. 24-31.

2. Davis,R., et al. SketchWizard Wizard of Oz Prototyping of Pen-Based User Interfaces.UIST’07, 119-128, ACM.

3. Duh, H.B., et al. Usability Evaluation for Mobile Device:A Comparison of Laboratory and Field Tests. MobileHCI'06.pp.181-186 ACM.

4. Hagen, P., et al. Emerging research methods for understanding mobile technology use.OZCHI'05, ACM.

5. Hartmann, B., et al. Wizard of Oz Sketch Animation for Experience Prototyping. Ubicomp 2006, 17-21, 2006.

6. Holmquist, L., Prototyping: generating ideas or cargo cult designs? Interactions, 2005. 12(2), ACM.

7. Hulkko,S.,et al.Mobile Probes.NordCHI'04,43-51,ACM.

8. Kjeldskov, J. and J. Stage, New Techniques for Usability Evaluation of Mobile Systems. International Journal of Human Computer Studies, Elsevier, 2003.

9. Klemmer, S., et al,SUEDE: A Wizard of Oz Prototyping Tool for Speech User Interfaces. UIST’00, 1-10, ACM.

10. Landay, J., SILK: Sketching Interfaces Like Crazy. CHI'96, pp.398-399, ACM.

11. Lin, J., et al, DENIM: Finding a Tighter Fit Between Tools and Practice for Web Site Design.CHI’00, pp. 510-517, ACM.

12. McCurdy, M., et al. Breaking the Fidelity Barrier. CHI’06, pp. 1233 - 1242, ACM.

13. Nielsen, C.M., et al. It's Worth the Hassle! The Added Value of Evaluating the Usability of Mobile Systems in the Field. NordiCHI’06, pp. 272 - 280, ACM.

14. de Sá, M. and Carriço, L. 2008. Lessons from early stages design of mobile applications.MobileHCI'08,pp.127-136, ACM.

15. de Sá, M. and Carriço, L. 2006. Low-fi prototyping for mobile devices. In CHI '06 Extended Abstracts, pp. 694-699, ACM.

16. de Sá, M., Carriço, L., Duarte, L., and Reis, T. 2008. A mixed-fidelity prototyping tool for mobile devices. In Proceedings AVI '08, pp. 225-232, ACM.

17.Liu, L., and Khooshabeh, P. Paper or interactive?: a study of prototyping techniques for ubiquitous computing environments. CHI '03 Extended abstracts, 1030-1031, ACM.

18.Lumsden, J., MacLean, R. "A Comparison of Pseudo-Paper and Paper Prototyping Methods for Mobile Evaluations. MONET'2008, LNCS OnTheMove (OTM), 2008. 